\title{
Surface plasmon resonance optical sensor for COVID-19 detection
}

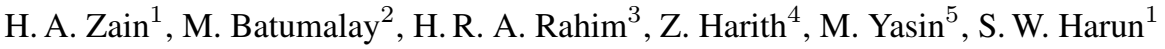 \\ ${ }^{1}$ Photonics Engineering Laboratory, Department of Electrical Engineering, University of Malaya, \\ 50603 Kuala Lumpur, Malaysia \\ ${ }^{2}$ Faculty of IT, INTI International University, 71800 Nilai, Negeri Sembilan, Malaysia \\ ${ }^{3}$ Faculty of Electronic and Computer Engineering, Universiti Teknikal Malaysia Melaka, Melaka, Malaysia \\ ${ }^{4}$ Faculty of Engineering and QS, INTI International University, 71800 Nilai, Negeri Sembilan, Malaysia \\ ${ }^{5}$ Department of Physics, Faculty of Science and Technology, Airlangga University, Surabaya, Indonesia
} swharun@um.edu.my

\begin{abstract}
The transmission of SARS-CoV-2, the novel severe acute respiratory syndrome corona virus have caused the corona virus disease (Covid-19) worldwide pandemic. Overcoming this pandemic requires identifying patients to avoid further spread of the disease. Real-time, sensitive, and costefficient methods for detecting the COVID-19 virus are crucial. Optical sensors provide one such means to achieve this, especially using surface plasmon resonance due to its advantages such as high sensitivity and excellent detection limits. In this paper, we propose a sensor for COVID-19 detection which is based on a simple Kretschmann configuration with gold layers and thiol-tethered DNA for the ligand layer. Angle interrogation was used to obtain the sensitivity of this structure using Matlab numerical analysis. The performance of the sensor was investigated with two types of prisms, SF10 and SF11, while varying the gold layer thickness between $45-60 \mathrm{~nm}$. This information was then used to determine which combination of prism and gold thickness are ideal for detecting COVID-19 using thiol-tethered DNA. Thiol-tethered DNA layer sensor showed the highest sensitivity of 137 degree/RIU when a SF10 prism was used with a 50-60 nm gold layer and thiol tethered DNA layer.
\end{abstract}

Keywords: optical sensors, COVID-19, surface plasmon resonance, plasmonic, energy efficiency.

Received: 19 July 2021

Revised: 9 October 2021

\section{Introduction}

Coronavirus detection is an urgent step in controlling the spread of the COVID-19 pandemic which caused loss of life and disruption of daily life. Identifying infected patients is needed to control the spread of this disease and mitigate its effects. However, the diverse clinical presentations of this disease such as cough, sore throat, diarrhea, and fever [1], make conclusive tests necessary. The long life of this virus on surfaces ranges from 2 - 72 hours [2] shows the continued need to detect the virus early and conclusively. Sensors that fulfill the ASSURED criteria are needed for COVID-19 control; ASSURED stands for affordability, sensitivity, specificity, user-friendliness, rapidity, and robustness, being equipment-free, and deliverability to end-users.

Current methods for detecting COVID-19 suffer from the need of complicated equipment and high cost. Some of the current technologies such as CT examinations show only pneumonia and lung lesions, which are a sign of COVID-19 but can also be caused by other diseases [3-5]. Enzyme-linked immunosorbent assay (ELISA) and reversetranscription polymerase chain reaction (RT-PCR) are more common tests, but they require multiple steps, trained professionals to conduct them, and have a long response time [6,7]. RT-PCR, currently the most common testing method; has been shown to give false-negatives before and needed specialized equipment and trained professionals to be performed [3]. This caused the RT-PCR test to be a bottleneck in the efforts to control the COVID-19 pandemic. Additionally, these tests are also expensive and require specialized delicate equipment. Thus, the challenge of detecting COVID-19 with inexpensive reliable equipment is still an unsolved problem.

Optical sensors might provide a potential solution to this challenge due to the improved sensitivities and detection limits of modern optical sensors, especially ones using surface plasmon resonance. Surface plasmon resonance optical sensors are label-free, efficient, and give real-time measurements. Optical sensors have been shown to have good selectivity, short response time, and can be optimized to be cost-beneficial [8-10]. Thus, thoroughly examining the potential of using surface plasmon resonance optical sensors is necessary. Surface plasmon resonance optical sensors use the surface plasmon resonance phenomenon and the resulting wavelength shift, angle shift, or intensity change to detect an analyte $[11,12]$. Surface plasmon waves are free-electron density waves in a thin metal surface such as gold or silver $[13,14]$. If p-polarized with the matching wave vector, these waves hit the metal boundary, these surface plasmon waves become excited and the light energy gets coupled into them causing the reflectivity of the metal surface 
to have a dip. The coupled light needs to match the wave vector of the polaritons and the way to achieve that is the use of a prism, fiber gratings, or D-shaped fiber evanescent mode [15-17].

If a Kretschmann setup is used for the sensor, the light hits the prism and achieves attenuated internal reflection, causing the evanescent mode to penetrate the thin metal coating layer on the prism. If the angle of incident light is the surface plasmon resonance angle, the wave vector of the light and the surface plasmon waves match, light is coupled into these waves. Thus, a dip appears in the light reflected from the prism. This angle is sensitive to changes in the refractive index of the medium surrounding the prism-metal interface, where the analyte is usually applied. Changes in the analyte refractive index, for instance, caused by the ligand and the analyte interacting on the sensor, cause changes in the surface plasmon resonance angle. By tracking these changes, the surface plasmon resonance Kretschmann setup can be used as a sensor [18].

Surface plasmon resonance sensors have been used before to detect many pathogens such as dengue [18, 19], H5N1 [20], Ebola [21], and hepatitis [22,23] virus surface antibodies. This shows the ability of surface plasmon sensors to detect pathogens in solutions. To detect a virus, there are multiple ways such as detection of elevated biomarkers like immunoglobin $\mathrm{M}(\mathrm{IgM})$ and immunoglobin $\mathrm{G}(\mathrm{IgG})$. A virus can be directly detected by the surface proteins. COVID-19 virus has $\mathrm{S}$ (spike), E (envelope), $\mathrm{M}$ (membrane), and $\mathrm{N}$ (nucleocapsids) proteins. For instance, similar diseases SARS-CoVand MERS-CoV have been detected using the viruses' proteins [24-26]. Viruses can also be detected by thiol tethered DNA. Surface plasmon resonance sensors have good potential to be used to detect the COVID-19 virus in samples.

Various optical methods have also been proposed and used to detect these viruses. For instance, photon counting has been used to improve the sensitivity of lateral flow assay technology. This technique can detect the density of antibodies with good sensitivity [27]. In other research, anano-plasmonic sensor for detecting SARS-CoV-2 monoclonal antibodies (mAbs) showed a detection limit of up to $370 \mathrm{vp} / \mathrm{mL}$ [28]. NP surface protein of the virus was also detected by immobilizing anti-SARS-CoV-2 antibodies on modified gold sensor chips [29]. Additionally, SPR imaging was also used to test the binding of IgM, IgG and IgA antibodies [30]. Plasmonic photothermal (PPT) effect and localized surface plasmon resonance (LSPR) with the help of two-dimensional gold nano islands (AuNIs) functionalized with complementary DNA receptors were also used to detect the virus [31]. Incorporating layers of silver, $\mathrm{BaTiO}_{3}$, and silicon have been shown to achieve sensitivity up to 130 degree/RIU in simulations [32]. In another simulation, gold nanorods (Au NRs) were used on BK7 prism to detect the virus spike protein with sensitivity of 111.11 degree/RIU [33]. In this work, a sensitivity $>120$ degree/RIU was reached in simulation. There is big potential for optical sensing of this virus.

In this paper, we present the preliminary design of a surface plasmon resonance sensor using the Kretschmann setup with gold thin layer coating and a thiol tethered DNA layer as a method to detect the COVID-19 in liquid samples. The theoretical and simulation work are our first step towards building this sensor in our laboratory and using the sensor to test actual COVID-19 sample collected from potential patients. These are basic but necessary steps towards developing an optical sensor for COVID-19.

\section{Working principle and sensor diagram}

In this surface plasmon resonance sensor, a Kretschmann prism configuration with multiple layers was used. To perform the numerical analysis, the transfer matrix method and Fresnel's equation were used [34]. This sensor has four layers: the prism, the thin gold layer, the thiol-tethered DNA, and lastly the test sample. In this work, the boundary condition at the first two layers $Z=Z_{1}=0$ and the last two layers have the boundary condition of $Z=Z_{n-1}$. The transfer matrix is the formula that relates the tangential components of the electric and magnetic fields for the first and last layers of the sensor and it is expressed in the following equation:

$$
\left[\begin{array}{l}
E_{1} \\
B_{1}
\end{array}\right]=M \times\left[\begin{array}{l}
E_{n-1} \\
B_{n-1}
\end{array}\right] .
$$

Given that $E_{1}$ and $B_{1}$ are the tangential components of the electric and magnetic field of the first layer. $E_{n-1}$ and $B_{n-1}$ are the tangential components of the electric and magnetic field of the $N$ and $N-1$ layer. The $M$ matrix presents the sensing structure's characteristics. The reflection coefficients also depend on $M$. Since surface plasmon resonance needs p-polarized light (or known as TM polarized light), the M matrix for p-polarized light is given as:

$$
M=\left(\prod_{k=2}^{N-1} M_{k}\right)=\left(\begin{array}{ll}
M_{11} & M_{12} \\
M_{21} & M_{22}
\end{array}\right),
$$




$$
\begin{gathered}
M_{k}=\left[\begin{array}{cc}
\cos \delta_{k} & \frac{-i \sin \delta_{k}}{\gamma_{k}} \\
-i \gamma_{k} \times \sin \delta_{k} & \cos \delta_{k}
\end{array}\right], \\
\gamma_{k}=\sqrt{\frac{\mu_{k}}{\epsilon_{k}}} \times \cos \theta_{k}=\frac{\sqrt{\epsilon_{k}-n_{1}^{2} \sin ^{2} \theta_{1}}}{\epsilon_{k}}, \\
\delta_{k}=\frac{2 \pi}{\lambda} n_{k} \cos \theta_{k}\left(Z_{k}-Z_{k-1}\right)=\frac{2 \pi d_{k}}{\lambda} \sqrt{\epsilon_{k}-n_{1}^{2} \sin ^{2} \theta_{1}} .
\end{gathered}
$$

Here, $\mu_{k}$ and $\epsilon_{k}$ are the magnetic permeability and the electric permittivity, respectively. $\lambda$ is the operating wavelength and $d_{k}$ is the thickness of the $k^{\text {th }}$ layer of the sensor system. The total reflection coefficient, $r_{p}$ will be:

$$
r_{p}=\frac{\left(M_{11}+M_{12} \gamma_{N}\right) \gamma_{1}-\left(M_{21}+M_{22} \gamma_{N}\right)}{\left(M_{11}+M_{12} \gamma_{N}\right) \gamma_{1}+\left(M_{21}+M_{22} \gamma_{N}\right)},
$$

with $M_{11}, M_{12}, M_{21}, M_{22}$ being the coefficients of the transfer matrix and the reflectivity is given as [32,34-36]:

$$
R_{p}=\left|r_{p}\right|^{2}
$$

The sensor system consists of three main components: He-Ne laser source, photo detector and prism as shown in Fig. 1. He-Ne laser source with $632.8 \mathrm{~nm}$ wavelength will be used to provide light [13] and a photo detector will be used to receive the light reflected from inside the prism and the reflectivity will be calculated by using Equations (1) to (7). All these calculations were done using a Matlab. The prism is a vital part of the sensor system and for this simulation, SF11 or SF10 were chosen. A very thin layer of gold (with a thickness ranging from 45 to $60 \mathrm{~nm}$ ) is deposited on the prism surface $[14,15,37,38]$. After that, the thiol-tethered DNA of the analyte (the virus) will be immobilized on the surface of that gold layer. The assumption is that the solution containing the analyte will be applied to the chip surface by a sealed flow cell to guarantee safety. The virus will take some time to bind with the thiol tethered DNA and cause a refractive index change on the prism surface. Previous work has shown that the thiol tethered DNA has a refractive index of $1.3-1.5$, depending on the layers' thickness. At a 4 nm thickness, the refractive index will approach $1.4[32,39]$.

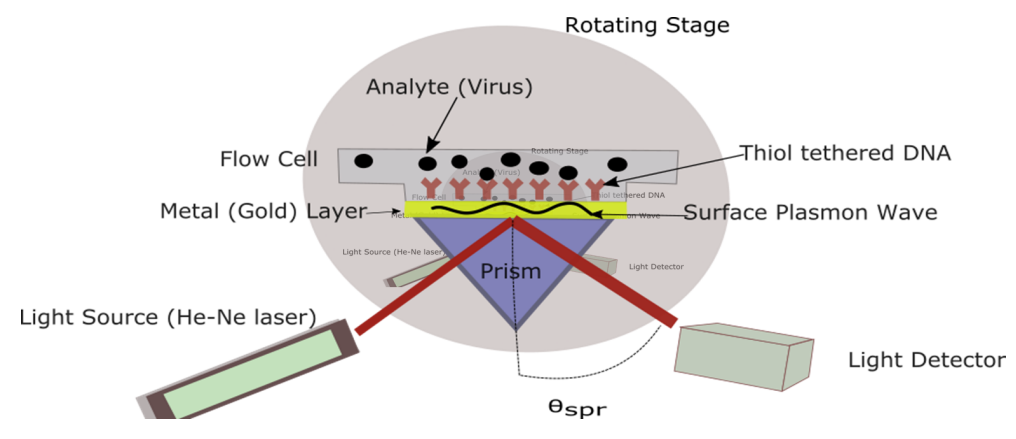

FIG. 1. The proposed sensor system

In this work, the average refractive index of the virus is estimated to be 1.54 , the membrane protein has a refractive index of $1.46 \pm 0.006$ while the virus load density in a liquid sample is estimated to be at average of $7 \times 10^{6}[29,33]$. If the virus exists in a sample applied to the coated prism surface, and as time passes, the binding will happen between the layer and the virus, causing a refractive index to change that results in a resonance angle shift. In the calculation, two values of refractive index are used: 1.33 for a clean aqueous sample and 1.34 to show the potential of the sensor to detect the binding between the virus in a sample and the sensing layer of the prism. The resonant angle at the minimum reflectivity is obtained in this work. The change in the resonant angle with the presence of the virus in the sample is investigated. It indicates the ability of this sensor to detect the existence of ananalyte (the virus) in a solution.

Several performance parameters such as sensitivity, full width at half maximum/minimum (FWHM), detection accuracy (DA), figure of merit (FOM) and minimum reflectivity $\left(R_{\min }\right)$ are investigated in this work. These parameters show the feasibility of using this biosensor to detect an analyte or virus in a sample. The sensitivity of the surface plasmon resonance optical sensor is defined as the ratio of the change in resonance angle to the change in the surrounding refractive index. The binding effect of the analyte with the immobilized ligand on the thin metal layer of the sensor results in a change of that layers' refractive index. By tracking that refractive index change and the resonance angle shift, the sensitivity of the sensors can be calculated. The center resonance angle is the location of the minimum 
optical power while the FWHM angle is the angle at which the output is $3 \mathrm{~dB}$ above the zero level. The quality factor is also related to FWHM by the following formula:

$$
\text { Q-factor }=\frac{\theta_{0}}{\text { FWHM }} \text {. }
$$

DA is the ratio of the total angle shift and the FWHM, andalso depends on the sensor's signal to noise ratio (SNR). The FOM of the sensor is defined as the ratio between the sensitivity and the FWHM. The minimum value of reflectivity which happens at the surface plasmon resonance angle can be referred to as $R_{\min }$. $R_{\min }$ shows how much of the incident light is coupled into the surface plasmon wave. Smaller $R_{\min }$ values indicate better momentum transfer.

\section{Results and discussion}

A performance comparison between SF10 and SF11 prisms at four different gold layer thicknesses is investigated using MatLab simulation to calculate the reflectivity of the prism setup as the thickness varies between $45-60 \mathrm{~nm}$. The main concern is the feasibility of this experiment with readily available setups. The sensor setup performance parameters need to be examined for every potential setup between the two prism types and the thickness variation for the gold layer with the thiol-tethered DNA. Table 1 shows the refractive index of the layers of the sensor.

TABLE 1. The refractive index of the sensor's layers

\begin{tabular}{|c|c|c|}
\hline Layer & Refractive index & Reference \\
\hline \hline SF10 & 1.72314 & {$[34,40]$} \\
\hline SF11 & 1.77860 & {$[34,41]$} \\
\hline Gold & $0.1726+i 3.4218$ & {$[34]$} \\
\hline $\begin{array}{c}\text { Thiol-tethered DNA } \\
\text { at 4 nm thickness }\end{array}$ & 1.40 & {$[32,39]$} \\
\hline
\end{tabular}

Firstly, the reflectivity response of the setup for the SF10 prism at the four determined gold thicknesses is investigated and the results are presented in Figs. 2(a-d) and Table 2. Fig. 2(a) shows the sensor response when the gold layer thickness is $45 \mathrm{~nm}$, the subsequent Figs. 2(b,c,d) show the response when the gold layer thickness is 50, 55, $60 \mathrm{~nm}$ respectively. This helps navigating our choices in the laboratory testing stage.

TABLE 2. The SF10 prism performance

\begin{tabular}{|c|c|c|c|c|}
\hline \multicolumn{5}{|c|}{ SF10 Prism } \\
\hline Gold thickness (nm) & Sensitivity $\left(^{\circ} / \mathbf{R I U}\right)$ & $\boldsymbol{R}_{\min }(\boldsymbol{\%})$ & FWHM $\left(^{\circ}\right)$ & FOM \\
\hline \hline 45 & 133 & 0.17 & 8.5 & 15.65 \\
\hline 50 & 136 & 0.003 & 6.7 & 20.30 \\
\hline 55 & 137 & 0.05 & 5.4 & 25.37 \\
\hline 60 & 137 & 0.15 & 5.9 & 23.22 \\
\hline
\end{tabular}

Figure 2 shows the response of the SF10 prism sensor to the presence of the analyte in a liquid solution. For the $50 \mathrm{~nm}$ gold layer, the reflectivity reaches its lowest value as shown in Fig. 2(b). While for $45 \mathrm{~nm}$ gold layer, it shows deep dips as shown in Fig. 2(a). The deepest dip with narrow FWHM is achieved with $55 \mathrm{~nm}$ gold thickness as shown in Fig. 2(c). As can be seen in Figs. 2(a,b), the minimum reflectivity of the sensor occurs when the gold layer thickness is between $45-50 \mathrm{~nm}$. This shows the gold coating with thickness between $45-50 \mathrm{~nm}$ is suitable to be combined with thiol-tethered DNA. The minimum reflectivity of the sensor is slightly higher when the gold layer thickness is $50-60 \mathrm{~nm}$ as shown in Figs. 2(c and d) and thus it is less suitable for further experimentation.

Table 2 summarizes the performance of the sensor with SF10 prism. As expected, the $50 \mathrm{~nm}$ gold layer has the lowest $R_{\min }$. Meanwhile, the sensitivity is above 100 for all four thicknesses with the layers of 55 and $60 \mathrm{~nm}$ show the highest sensitivity of 137. Fig. 3 shows the sensitivity and figure of merit of the SF10 sensor as a function of 

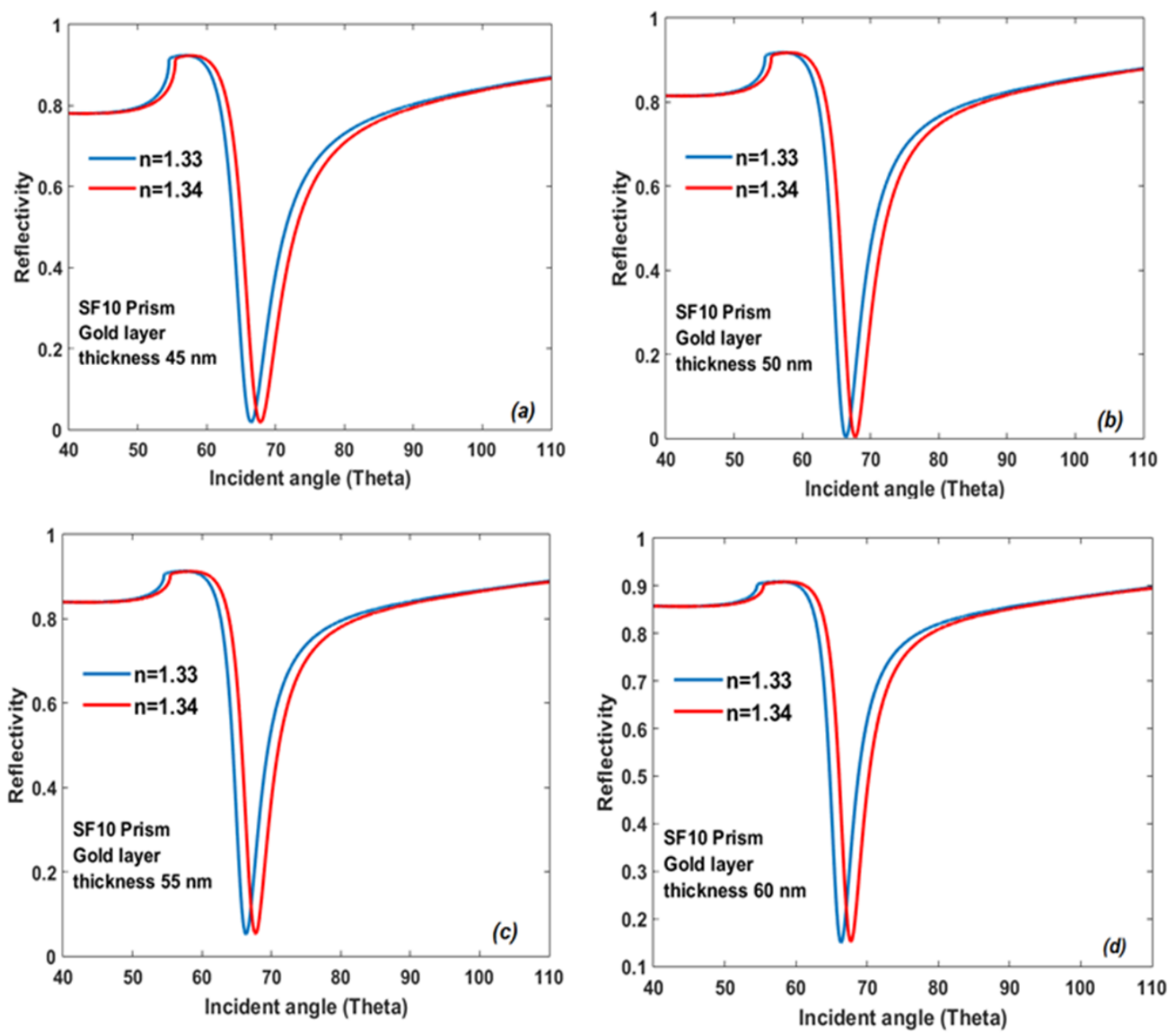

FIG. 2. The SF10 prism sensor response to the analyte with different gold layer thicknesses of (a) $45 \mathrm{~nm}$, (b) $50 \mathrm{~nm}$, (c) $55 \mathrm{~nm}$, and (d) $60 \mathrm{~nm}$
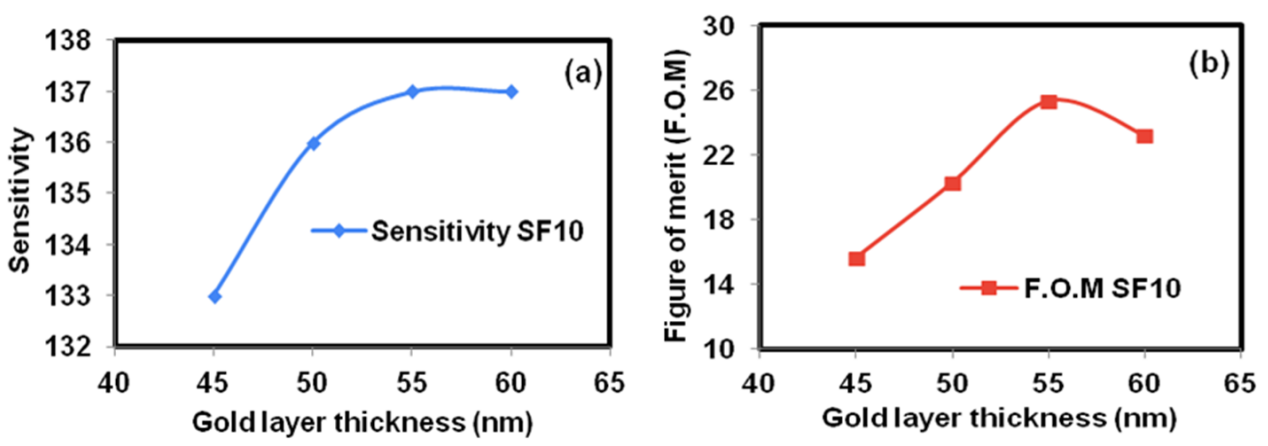

FIG. 3. The sensitivity and FOM against the thickness of gold layer for the sensor with SF10 prism

the gold layer thickness. Choosing the ideal sensor setup will be a trade-off between reflectivity, FOM and FWHM performances. If the best FOM is needed, then better option is to proceed with a 50-60 nm layer with an SF10 prism.

Figure 4 shows the sensor's response when an SF11 prism is used. This prism has a higher refractive index but can be costly, so a comparison between them and SF10 is necessary to decide which is better for detecting the analyte using thiol tethered DNA. Figs. 4(a,b,c and d) show the sensor response when the gold layer thickness is exactly 45, 50, 55, $60 \mathrm{~nm}$ respectively. Similar resonance dip and FWHM patterns are obtained as compared to the previous 
sensor with SF10 prism. However, the FWHM for the SF10 sensor was smaller. As can be seen in Fig. 4, the smaller reflectivity of the sensor occurs at the resonance angle when the gold layer thickness is between 45 and $50 \mathrm{~nm}$ as compared to the thicker thicknesses of 55 and $60 \mathrm{~nm}$. This indicates that the gold coating with thickness between $45-50 \mathrm{~nm}$ is suitable to be combined with thiol-tethered DNA for sensing application.
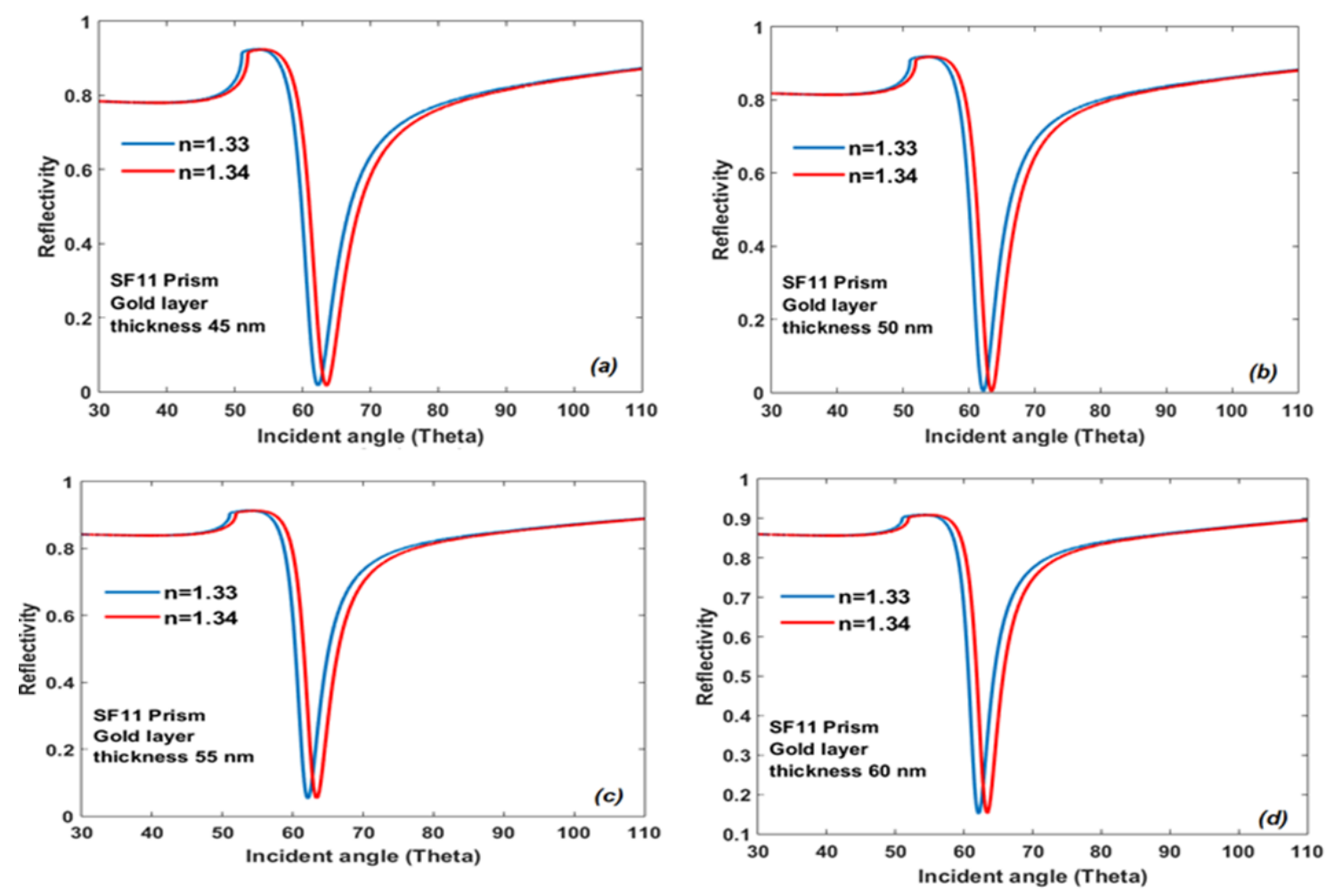

FIG. 4. The SF11 sensor performance with different gold layer thicknesses a) gold layer thickness $45 \mathrm{~nm}, \mathrm{~b}$ ) gold layer thickness $50 \mathrm{~nm}$, c) gold layer thickness $55 \mathrm{~nm}$, d) gold layer thickness $60 \mathrm{~nm}$

Figure 5 shows the sensitivity and FOM for the SF11 sensor. For this prism, the thiol-tethered DNA sensor has a lower but more stable sensitivity compared to the previous SF10 prism. The sensitivity varies within 125 to $126^{\circ} / R I U$ as the gold layer thickness was changed from 45 to $60 \mathrm{~nm}$. However, the FOM peaks at $55 \mathrm{~nm}$. Table 3 summarizes the performance of the sensor with SF11 prism. It indicates a similar performance with the previous prism.
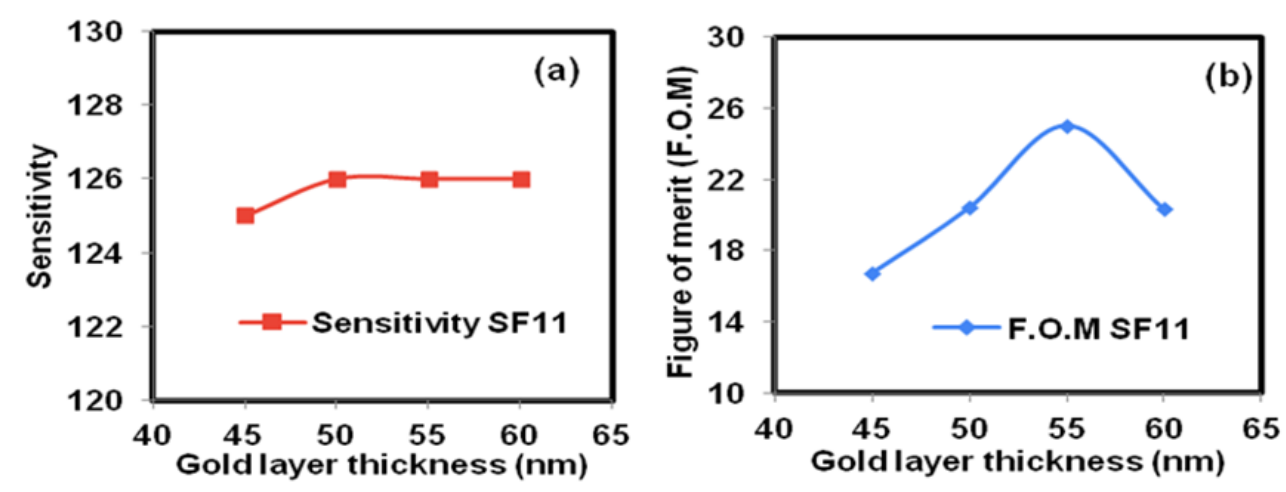

FIG. 5. (a) Sensitivity and (b) figure of merit against the gold thickness for the SF11 sensor 
TABLE 3. The SF11 sensor performance

\begin{tabular}{|c|c|c|c|c|}
\hline \multicolumn{5}{|c|}{ SF11prism } \\
\hline $\begin{array}{c}\text { Metal } \\
\text { thickness (nm) }\end{array}$ & $\begin{array}{c}\text { Sensitivity } \\
\text { SF11 }\left({ }^{\circ} / \text { RIU) }\right.\end{array}$ & $\boldsymbol{R}_{\text {min }}(\%)$ & FWHM $\left(^{\circ}\right)$ & F.O.M \\
\hline \hline 45 & 125 & 0.016 & 7.440 & 16.801 \\
\hline 50 & 126 & 0.003 & 6.160 & 20.455 \\
\hline 55 & 126 & 0.054 & 5.030 & 25.050 \\
\hline 60 & 126 & 0.153 & 6.180 & 20.388 \\
\hline
\end{tabular}

\section{Conclusion}

In this paper, a COVID-19 sensor using thiol-tethered DNA as a ligand layer with two types of prisms; SF10 and SF11 is proposed. Numerical analysis was completed using MATLAB, to study the response of the sensor. The aim was to determine the type of prism and the thickness of the gold layer which is ideal for thiol-tethered DNA detection of Covid-19. The two prisms were found to have comparable performance. However, SF10 prisms with gold coating between $50-60 \mathrm{~nm}$ demonstrated a superior performance in terms of sensitivity. Future work will focus on optimizing the layers to improve the FWHM and FOM of the proposed design.

\section{Acknowledgements}

This work was supported by the Airlangga University Research Grant (2021) and the University of Malaya (Grant No: ST036-2020).

\section{References}

[1] MenniC., Valdes A.M., et al. Real-time tracking of self-reported symptoms to predict potential COVID-19. Nature Medicine, 2020, 26 (7), P. 1037-1040.

[2] Van Doremalen N., Bushmaker T., et al. Aerosol and Surface Stability of SARS-CoV-2 as Compared with SARS-CoV-1. New England Journal of Medicine, 2020, 382 (16), P. 1564-1567.

[3] Xie X., Zhong Z., et al. Chest CT for Typical Coronavirus Disease 2019 (COVID-19) Pneumonia: Relationship to Negative RT-PCR Testing. Radiology, 2020, 296 (2), E41-E45.

[4] Li X., Zeng W., et al. CT imaging changes of corona virus disease 2019(COVID-19): a multi-center study in Southwest China. Journal of Translational Medicine, 2020, 18 (1), 154.

[5] Fang Y., Zhang H., et al. Sensitivity of Chest CT for COVID-19: Comparison to RT-PCR. Radiology, 2020,296 (2), E115-E117.

[6] Nguyen T., Duong Bang D., Wolff A. 2019 Novel Coronavirus Disease (COVID-19): Paving the Road for Rapid Detection and Point-of-Care Diagnostics. Micromachines (Basel), 2020, 11 (3), 306.

[7] Kaur M., Tiwari S., Jain R. Protein based biomarkers for non-invasive Covid-19 detection. Sens Biosensing Res, $2020,29,100362$.

[8] Jing J.-Y., Wang Q., Zhao W.-M., Wang B.-T. Long-range surface plasmon resonance and its sensing applications: A review. Optics and Lasers in Engineering, 2019, 112, P. 103-118.

[9] Lukose J., Chidangil S., George S.D. Optical technologies for the detection of viruses like COVID-19: Progress and prospects. Biosensors and Bioelectronics, 2021, 178, 113004.

[10] Amendola V., Pilot R., et al. Surface plasmon resonance in gold nanoparticles: a review. J. Phys. Condens. Matter, 2017,29 (20), 203002.

[11] Drobysh M., Ramanaviciene A., Viter R., Ramanavicius A. Affinity Sensors for the Diagnosis of COVID-19. Micromachines (Basel), 2021, 12 (4), 390.

[12] Abid S.A., Ahmed Muneer A., et al. Biosensors as a future diagnostic approach for COVID-19. Life Sciences, $2021,273,119117$.

[13] Prabowo B.A., Purwidyantri A., Liu K.C. Surface Plasmon Resonance Optical Sensor: A Review on Light Source Technology. Biosensors (Basel), 2018, 8 (3), 80.

[14] Gupta G., Kondoh J. Tuning and sensitivity enhancement of surface plasmon resonance sensor. Sensors and Actuators B: Chemical, 2007, 122 (2), P. 381-388.

[15] Sharma A.K., Jha R., Gupta B.D. Fiber-Optic Sensors Based on Surface Plasmon Resonance: A Comprehensive Review. IEEE Sensors Journal, 2007, 7 (8), P. 1118-1129.

[16] Roh S., Chung T., Lee B. Overview of the characteristics of micro- and nano-structured surface plasmon resonance sensors. Sensors (Basel), 2011, 11 (2), P. 1565-1588.

[17] Patil P.O., Pandey G.R., et al. Graphene-based nanocomposites for sensitivity enhancement of surface plasmon resonance sensor for biological and chemical sensing: A review. Biosensors and Bioelectronics, 2019, 139, 111324.

[18] Omar N.A.S., Fen Y.W., et al. Quantitative and selective surface plasmon resonance response based on a reduced graphene oxide-polyamidoamine nanocomposite for detection of dengue virus e-proteins. J. Nanomaterials, 2020,10 (3), 569.

[19] Jahanshahi P., Zalnezhad E., Sekaran S.D., Adikan F.R. Rapid immunoglobulin M-based dengue diagnostic test using surface plasmon resonance biosensor. Scientific Reports, 2014, 4, 3851.

[20] Bai H., Wang R., et al. A SPR aptasensor for detection of avian influenza virus H5N1. Sensors (Basel), 2012,12 (9), P. $12506-12518$. 
[21] Wang S., Shan X., et al. Label-free imaging, detection, and mass measurement of single viruses by surface plasmon resonance. Proceedings of the National Academy of Sciences of the USA, 2010, 107 (37), P. 16028-16032.

[22] Uzun L., Say R., Unal S., Denizli A. Production of surface plasmon resonance based assay kit for hepatitis diagnosis. Biosensors and Bioelectronics, 2009, 24 (9), P. 2878-2884.

[23] Tam Y.J., Zeenathul N.A., et al. Wide dynamic range of surface-plasmon-resonance-based assay for hepatitis B surface antigen antibody optimal detection in comparison with ELISA. Biotechnology and Applied Biochemistry, 2017, 64 (5), P. 735-744.

[24] Zhao G., He L., et al. A novel nanobody targeting Middle East respiratory syndrome coronavirus (MERS-CoV) receptor-binding domain has potent cross-neutralizing activity and protective efficacy against MERS-CoV. Journal of Virology, 2018,92 (18), e00837-18.

[25] Ahn D.G., Jeon I.J., et al. RNA aptamer-based sensitive detection of SARS coronavirus nucleocapsid protein. Analyst, 2009, 134 (9), P. 18961901.

[26] Chandra S., Bharadwaj R., Mukherji S. Label free ultrasensitive optical sensor decorated with polyaniline nanofibers: Characterization and immunosensing application. Sensors and Actuators B: Chemical, 2017, 240, P. 443-450.

[27] Peng T., Liu X., et al. Enhancing sensitivity of lateral flow assay with application to SARS-CoV-2. Applied Physics Letters, 2020 , 117 (12), 120601.

[28] Huang L., Ding L., et al. One-step rapid quantification of SARS-CoV-2 virus particles via low-cost nanoplasmonic sensors in generic microplate reader and point-of-care device. Biosensors and Bioelectronics, 2021, 171, 112685.

[29] Bong J.-H., Kim T.-H., et al. Pig Sera-derived Anti-SARS-CoV-2 Antibodies in Surface Plasmon Resonance Biosensors. BioChip Journal, 2020, 14 (4), P. 358-368.

[30] Schasfoort R.B.M.,van Weperen J., et al. High throughput surface plasmon resonance imaging method for clinical detection of presence and strength of binding of IgM, IgG and IgA antibodies against SARS-CoV-2 during CoViD-19 infection. Methods X, $2021, \mathbf{8}, 101432$.

[31] Qiu G., Gai Z., et al. Dual-functional plasmonic photothermal biosensors for highly accurate severe acute respiratory syndrome coronavirus 2 detection. ACS nano, 2020, 14 (5), P. 5268-5277.

[32] Uddin S.M.A., Chowdhury S.S., Kabir E. Numerical Analysis of a Highly Sensitive Surface Plasmon Resonance Sensor for SARS-CoV-2 Detection. Plasmonics, 2021, P. 1-13.

[33] Das C.M., Guo Y., et al. Gold Nanorod Assisted Enhanced Plasmonic Detection Scheme of COVID-19 SARS-CoV-2 Spike Protein. Advanced theory and simulations, 2020, 3 (11), 2000185.

[34] Yamamoto M. Surface plasmon resonance (SPR) theory: tutorial. Review of Polarography, 2002, 48 (3), P. $209-237$.

[35] Rahman M.S., Anower M.S., et al. Design and numerical analysis of highly sensitive Au-MoS2-graphene based hybrid surface plasmon resonance biosensor. Optics Communications, 2017, 396, P. 36-43.

[36] Saifur Rahman M., Rikta K.A., Bashar L.B., Anower M.S. Numerical analysis of graphene coated surface plasmon resonance biosensors for biomedical applications. Optik, 2018, 156, P. 384-390.

[37] Brahmachari K., Ray M. Effect of prism material on design of surface plasmon resonance sensor by admittance loci method. Frontiers of Optoelectronics, 2013, 6 (2), P. 185-193.

[38] Sharma N.K., Yadav S., Sajal V. Theoretical analysis of highly sensitive prism based surface plasmon resonance sensor with indium tin oxide. Optics Communications, 2014, 318, P. 74-78.

[39] Peterlinz K.A., Georgiadis R.M., Herne T.M., Tarlov M.J. Observation of Hybridization and Dehybridization of Thiol-Tethered DNA Using Two-Color Surface Plasmon Resonance Spectroscopy. Journal of the American Chemical Society, 1997, 119 (14), P. 3401-3402.

[40] SCHOTT, Optical Glass Collection N-SF10, in 728284.428, ed, 2014, URL: https://shop.schott.com/advanced_optics/en/Optical-Glass/NSF10/c/optical-glass/glass-N-SF10.

[41] SCHOTT, Optical Glass Collection N-SF11, in 785257.322, ed, 2014, https://shop.schott.com/advanced_optics/en/N-SF11/c/glass-N-SF11. 